\title{
Treatment of Nonsense Mutations Using Stop Codon Read-through Therapeutics and Creation of Animal Models Using CRISPR-Cas9
}

\author{
Kelly A. Turner ${ }^{1,2,3}$ and Francis YM Choy ${ }^{1^{*}}$ \\ ${ }^{1}$ Centre for Biomedical Research, Department of Biology, University of Victoria, 3020, Station CSC, Victoria, BC, V8W 3N5, Canada \\ ${ }^{2}$ Terry Fox Laboratory, British Columbia Cancer Agency, Vancouver, BC, Canada V5Z 1 L3 \\ ${ }^{3}$ Department of Medical Genetics, University of British Columbia, Vancouver, BC, Canada V6T $1 Z 3$
}

*Corresponding author: Francis Y.M. Choy, Centre for Biomedical Research, Department of Biology, University of Victoria, 3020, Station CSC, Victoria, BC, V8W 3N5, Canada, Tel: 1-250-721-7107; Fax: 1-250-721-7120; E-mail: fchoy@uvic.ca

Received date: May 23, 2015; Accepted date: June 27, 2015; Published date: July 04, 2015

Copyright: (c) 2015 Turner KA, et al. This is an open-access article distributed under the terms of the Creative Commons Attribution License, which permits unrestricted use, distribution, and reproduction in any medium, provided the original author and source are credited.

\begin{abstract}
Premature termination codons result when a mutation within a protein coding sequence causes an amino acidencoding sense codon to be interpreted as a stop codon. Often, this results in truncation and destruction of the mRNA transcript or a non-functional protein. Nonsense mutations account for approximately $11 \%$ of all described mutations that contribute to disease. A number of therapeutics, including suppressor tRNAs, gentamicin, and PTC124, have been developed that purport to promote the "read-through" of these premature termination codons so that they are interpreted as sense codons. This, theoretically, would reconstitute the full-length transcript and restore protein/enzyme function and ameliorate disease symptoms. Currently, the implementation of such therapeutics in a clinical setting has been slow due to factors such as toxicity and inefficiency. We will discuss these hurdles as well as the difficulties associated with determination of the required protein/enzyme level to reduce symptoms as well as breakthroughs in genome editing to create nonsense mutation animal models using the clustered regularly interspersed short palindromic repeats-CRISPR-associated protein 9 system.
\end{abstract}

Keywords: Stop codon; Nonsense mutation; Suppressor tRNA; Gentamicin; PTC124; Read-through; CRISPR-Cas9

\begin{abstract}
Abbreviations:
Cas: CRISPR-associated; CK: creatine kinase; CRISPR: clustered regularly interspersed short palindromic repeats; crRNA: CRISPR RNA; DMD: Duchenne muscular dystrophy; FLuc: firefly luciferase; HDR: homology directed repair; iPSC: induced pluripotent stem cell; MKOS: c-Myc, Klf4, Oct4, Sox2 transcription factors; MPS-I-H: Mucopolysaccharidosis type I - Hurler's Syndrome; NHEJ: nonhomologous end joining; NMD: nonsense-mediated mRNA decay; PAM: protospacer adjacent motif; PTC: premature termination codon
\end{abstract}

\section{Introduction}

The central dogma of molecular biology states that the genetic code in the form of DNA is transcribed into RNA, which is then translated into protein [1]. The translation of RNA to protein involves the interpretation of three letter codons that correspond to amino acids. The interpretation of the code and the linear assembly of amino acids are facilitated by the ribosomal machinery. Subsequently, the primary amino acid sequence gets processed and folds into the mature protein. If a mutation occurs within the sequence so that an amino acidencoding sense codon is mistakenly interpreted as a stop codon, translation is halted and this results in a truncated and dysfunctional protein; in other cases, the transcript is prematurely degraded via nonsense-mediated mRNA decay (NMD), a post-transcriptional quality control process in eukaryotes that degrades transcripts carrying disease-causing premature termination codons (PTCs) [2]. Nonsense mutations account for $11 \%$ of all described mutations that contribute to disease [3]. Consequently, if treatments are available that allow for the "read through" of these PTCs, that is, if the stop codon is interpreted as a sense codon, then the correct sequence should be reconstituted and a full-length, functional protein will result. Treatments that facilitate the read through of PTCs have the potential to treat a genotypic subset of patients with such a defect, regardless of the disease [4]. Currently, the implementation of such treatments in a clinical setting has been unsuccessful due to a number of factors. Difficulties with three particular treatments - suppressor tRNAs, gentamicin, and PTC124 will be discussed as well as general hurdles including the difficulty in creating animal models and the determination of the required threshold of reconstituted protein activity to ameliorate symptoms.

\section{Specific Treatments}

\section{Suppressor tRNAs}

Historically, the use of suppressor tRNAs was the first method that demonstrated read through ability in $\beta$-thalassemia patients with nonsense mutations [5]. $\beta$-thalassemia is an autosomal recessive disorder that results from mutations in the $\beta$-globin locus on chromosome 11q15.5, which lead to profoundly deficient hemoglobin synthesis and anemia [6]. Temple et al. designed a tRNA anticodon for lysine with two mismatches in the recognition sequence so that a specific stop codon (UAG) became recognized as a lysine codon. This was referred to as a suppressor tRNA. Subsequently, if this stop codon was encountered, the suppressor tRNA would incorporate a lysine at that site instead of terminating translation. Xenopus oocyte nuclei were injected with a plasmid containing the modified tRNA ${ }^{\text {Lys }}$ gene and later injected with reticulocyte mRNA from a $\beta$-thalassemia patient with a lysine to UAG nonsense mutation. Importantly, the 
mutated tRNA ${ }^{\text {Lys }}$ would not recognize the natural stop codon in $\beta$ globin as it has a UAA sequence. The oocytes were incubated for 48 hours and the $\beta$-globin protein was isolated using a monoclonal antibody. When the protein was run on a gel, it had the same electrophoretic mobility as normal $\beta$-globin, suggesting that the incorporation of lysine at the premature stop codon reconstituted the full-length protein.

In vivo studies in mice modeling Duchenne muscular dystrophy (DMD) were later carried out and the mice showed a $2.5 \%$ increase in dystrophin expression in muscle fibers when injected with suppressor tRNAs [7]. These findings suggested that human genetic diseases caused by nonsense mutations may also be amenable to treatment using such an approach. However, the usual roadblocks associated with gene therapy have impeded a clinical application of suppressor tRNAs. Various gene delivery systems such as retroviral and adenoviral transfection as well as liposomal packaging have been described, but each are associated with the drawbacks of insertional mutagenesis [8], immune rejection [9], and toxicity [10], respectively. Additionally, assuming that the suppressor tRNA gene is actually expressed, the mature tRNA must then be recognized and charged by specific aminoacyl synthetases [11]. The modification of the tRNA may result in decreased recognition and inefficient charging by the aforementioned enzymes, which would lead to a decrease in readthrough. An additional hurdle for the exogenous suppressor tRNA is competition with release factors that recognize the PTC and catalyze the cleavage of the polypeptide from the ribosome [12]. Importantly, suppressor tRNAs are unable to distinguish between natural stop codons and premature stop codons, which could have disastrous consequences for normal protein translation. Currently, the implementation of suppressor tRNAs as a therapy for PTC readthrough does not appear to be a viable option until gene delivery is improved and safety concerns associated with natural stop codon readthrough are addressed.

\section{Gentamicin}

A pharmaceutical compound that shows nonsense suppression activity is the aminoglycoside gentamicin. Aminoglycosides are a class of antibiotics that selectively bind to the bacterial ribosomal decoding center, which leads to misincorporation of amino acids in the growing peptide chain and improper protein synthesis [13]. A subset of aminoglycosides, including gentamicin, occasionally bind to eukaryotic ribosomes as well [14], which leads to misincorporation of amino acids at PTCs, but not at natural stop codons. The selectivity of gentamicin for promoting read through at PTCs but not at natural stop codons is not well-understood, although it was proposed that the increased distance between the PTC and polyA-binding protein of the mRNA closed loop complex creates a lag time whereupon a charged tRNA can accidentally enter the ribosomal A-site and continue synthesis of the polypeptide chain [15] (Figure 1a and b).

The first in vivo application of stop codon read-through using gentamicin was in mdx mice modeling DMD [16]. DMD is an Xlinked muscular degenerative disorder that results from a profound deficiency or absence of the dystrophin protein in muscle fibers [17]. Data obtained from The Human Gene Mutation Database suggests that nonsense mutations account for $20 \%$ of all mutational events leading to DMD [3]. Subsequently, nonsense suppression therapies could benefit a large subset of DMD patients. In the pioneering in vivo experiment, $\mathrm{mdx}$ mice were treated with gentamicin for 14 days and subsequently analyzed for creatine kinase (CK) activity (upregulated during muscle death) and full-length dystrophin incorporation into muscles. The mdx mice treated with gentamicin had decreased creatine kinase activity compared to untreated $\mathrm{mdx}$ mice, with levels approaching those of normal control mice. As well, western blot analysis of dystrophin in muscles using an antibody directed against the C-terminal-region of the dystrophin protein showed a significant increase in the amount of full-length dystrophin present in muscles compared to the untreated mice. Overall, treatment with gentamicin was able to restore $10-20 \%$ of normal dystrophin levels in muscle of $\mathrm{mdx}$ mice, which provided some protection against muscle injury but did not restore full function of the muscles. The authors postulated that daily administration of gentamicin in humans (below the maximum recommended dose) could provide some increase in dystrophin levels in patients with DMD.

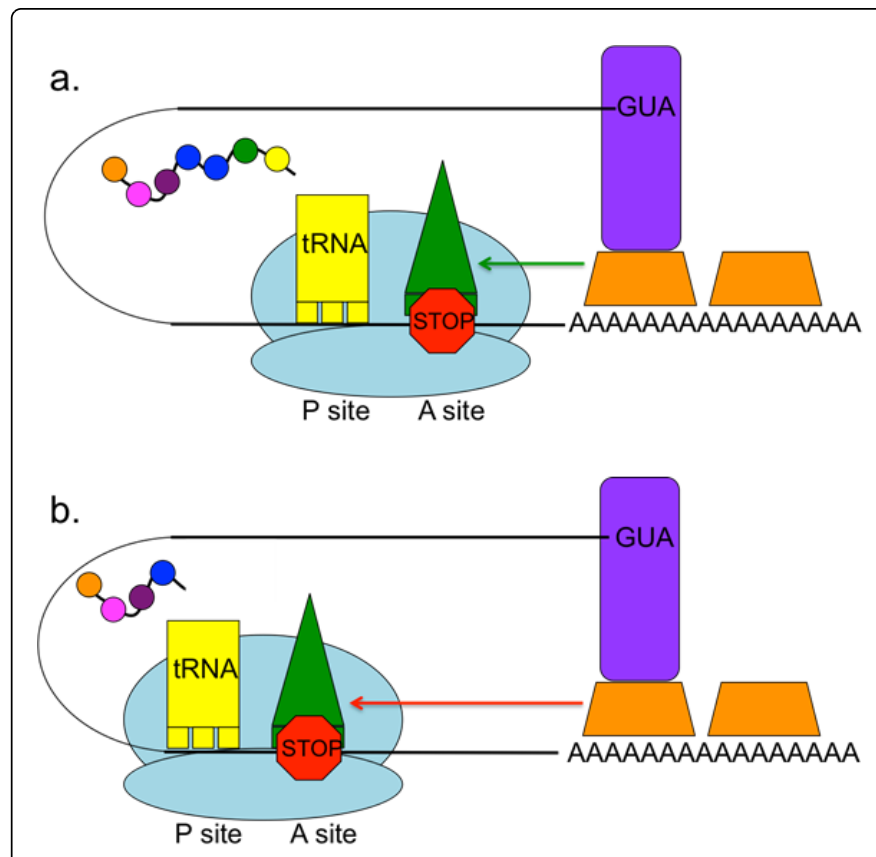

Figure 1: Translation termination at normal versus premature stop codons. a) Normal translation termination. Release factors (green triangle) bind to the A site of the ribosome and through interactions with poly(A) binding proteins (orange trapezoid), facilitate cleavage of the polypeptide chain. b) Translation termination at PTCs. The release factors and poly(A) binding proteins are farther apart (red arrow), creating a lag time in which the PTC may be susceptible to read-through. This property can be enhanced by nonsense suppression drugs.

Building on the results from the murine model, Wagner et al. treated human DMD patients with sub-toxic levels of gentamicin for two weeks. At the end of the trial period, no full-length dystrophin was detected in any of the patients; however, serum CK levels had decreased, which seemed contradictory as CK activity is used as a biomarker for damaged muscle cells and a proxy for diagnosing DMD. A decrease in CK would indicate that dystrophin levels had increased [18]. Wagner et al. postulated that the discrepancy was due to less daily activity in patients during the experiment and that CK levels are not an accurate marker of disease. They recommended that higher dosages of gentamicin should be used in future studies for longer periods of time; however, they cautioned that dose escalation could lead to 
nephrotoxicity and ototoxicity. Their alternative recommendation is to explore other aminoglycosides that are less toxic or use a rational design approach to develop similar molecules that demonstrate readthrough ability, but are non-toxic.

A recent in vitro study examined the use of gentamicin on PTC skin fibroblasts from a subset of patients with xeroderma pigmentosum, a severe skin condition caused by mutations in $X P C$, which encodes an enzyme associated with DNA repair following ultraviolet radiation exposure. Following gentamicin treatment, the amount of $X P C$ mRNA as well as $X P C$ protein significantly increased. The authors believe that even a small increase in read-through (3-5\% of normal $X P C$ mRNA and $29 \%$ of normal XPC protein) may decrease the risk of skin cancer for these patients, and topical delivery of the drug may circumvent the concerns associated with aminoglycoside toxicity and provide a method of personalized medicine to alleviate the cutaneous symptoms [19].

\section{PTC124}

Another pharmacological compound that may demonstrate PTC read-through ability is PTC124 (Ataluren; 3-[5-(2-fluorophenyl)-1,2,4oxadiazol-3-yl]benzoic acid). PTC124 was discovered from a highthroughput screen of approximately 800,000 compounds using a firefly luciferase (FLuc) activity assay system [20]. A construct was created in which the luciferase gene contained an in-frame PTC. Human embryonic kidney cells were then transfected with the construct. Theoretically, if a compound could successfully suppress the PTC, then the full-length luciferase transcript would be translated and increased fluorescence could be detected. The candidate compound also had to fulfill other requirements such as high oral bioavailability, low toxicity, and minimal off-target interactions in order to be considered for further investigation. PTC124 showed all of these characteristics and had the ability to suppress PTCs in human DMD primary cell cultures and $\mathrm{mdx}$ mice, thereby increasing dystrophin levels in vitro and in vivo and ameliorating disease symptoms in the mice, such as contractioninduced muscle injury, which is the major functional deficit in $\mathrm{mdx}$ mice as well as DMD patients [20].

The success of PTC124 in mdx mice prompted clinical trials for the drug in human DMD patients. Unfortunately, in the phase IIb clinical trial, no significant changes were observed between the experimental and placebo groups over the 48-week period and the trial was discontinued [21]. As other laboratories attempted to replicate the results obtained by Welch et al. on other cell lines, conflicting results began to emerge. In order to determine whether the up-regulation of the luciferase signal was due to PTC suppression or an artifact of the assay, various hypotheses were proposed that could explain the results obtained by Welch et al. [22,23]. Experiments performed by Auld et al. demonstrated that PTC124 did not promote PTC read-through, but simply stabilized the few full-length luciferase transcripts that arose from natural, "leaky" nonsense suppression, thus resulting in a significant increase in fluorescence over the background. Also, when a structurally dissimilar reporter enzyme was used (Renilla reniformis luciferase, or RLuc), no fluorescence was detected [22]. The authors suggested that appropriate control experiments should have been carried out, which could have determined that FLuc is a molecular target of PTC124 and not a suitable reporter for this class of compound.

Despite the controversial results of the FLuc assay, PTC124 has been used in a number of in vitro studies of very different diseases resulting from nonsense mutations, including cystic fibrosis (CF) [24],
Mucopolysaccharidoses types I and VI [25,26], pulmonary aterial hypertension [27], and methylmalonic aciduria [28], all with positive results. Since these deficient proteins are functionally diverse, it is unlikely that the mechanism of restoration seen in the FLuc assay (binding and subsequent stabilization of the enzyme) was the same mechanism employed to restore the different proteins associated with the aforementioned diseases.

Furthermore, PTC124 has been used in clinical trials with CF patients, and phase III data suggested that PTC124 improved several symptom parameters relative to controls, however, the restored protein function was not significant enough to have a therapeutic benefit [29]. The small increase in protein function suggests that PTC124 may be effective in treating diseases in which the required protein threshold is low, but few studies to date have explored such disorders.

\section{General Hurdles}

The application of the aforementioned therapies as well as other proposed treatments for PTC read-through in the clinic has been slow due to not only intrinsic shortcomings of the therapies themselves, but also the difficulty of modeling the diseases in animals. Another significant hurdle is the large variability in the level of protein/enzyme activity that is required to alleviate disease symptoms; these are often disease-specific, patient-specific, and depend upon the context of the mutation. Upon closer scrutiny, the seemingly 'cure-all' nature of these drugs against diseases caused by premature stop codons is a hasty assumption as the technology is still in its infancy, and a number of obstacles still remain.

\section{Animal models}

A significant factor that has stalled the implementation of PTC suppression therapy is the lack of animal models carrying the precise nonsense mutation associated with the various diseases. Currently, knockout animal models are costly and take a minimum of several months to produce, and conventional methods knock out large portions of the gene of interest [30]. It is more difficult to introduce a single nucleotide substitution at a precise location in the genome that creates an in-frame premature stop codon. However, a newly emerging technique known as CRISPR-Cas (clustered regularly interspaced short palindromic repeats-CRISPR-associated protein) co-opts an endogenous prokaryotic defense mechanism that uses RNA to guide the degradation of foreign DNA [31]. This CRISPR-Cas system can be used to create double stranded DNA breaks at very precise locations in the genome and allow for subsequent non-homologous end joining (NHEJ) gene disruption to create small indels, or precise editing via homology directed repair (HDR), if a modified DNA oligo complementary to the cleaved locus is present [32] (Figure 2). For example, it is possible to introduce a base substitution at a precise locus in the genomic sequence that converts a sense codon into an in-frame PTC.

The aforementioned method was applied to create precise mutations in mouse embryos with high efficiency and in significantly less time (a few weeks instead of many months) compared to conventional methods [33]. Zygotes were co-injected with guide CRISPR RNAs (crRNAs) complementary to a specific region in the Tet 1 gene, Cas 9 mRNA, and single stranded DNA oligos that would serve as a template for HDR once the target locus was cleaved. The DNA oligo was designed with two base changes so that a SacI restriction site was converted to an EcoRI site. Blastocysts were derived from these zygotes 
and the DNA was isolated and subsequently amplified and digested with EcoRI to detect whether the oligo facilitated HDR. Six out of nine embryos incorporated an EcoRI site at the Tet 1 locus. These blastocysts were injected into a surrogate female and $21 \%$ developed to term and resulted in viable offspring [33]. As an interesting aside, Wang et al. were also able to induce the simultaneous disruption of five genes in mouse embryonic stem cells in a single step using this same method, but with 5 different crRNAs and five different DNA oligos specific to the genes of interest. After restriction fragment length polymorphism analysis, it was determined that $10 \%$ of the clones carried mutations in all five genes. The results obtained by Wang et al. demonstrate that it is no longer difficult to model diseases that result from very specific mutations.
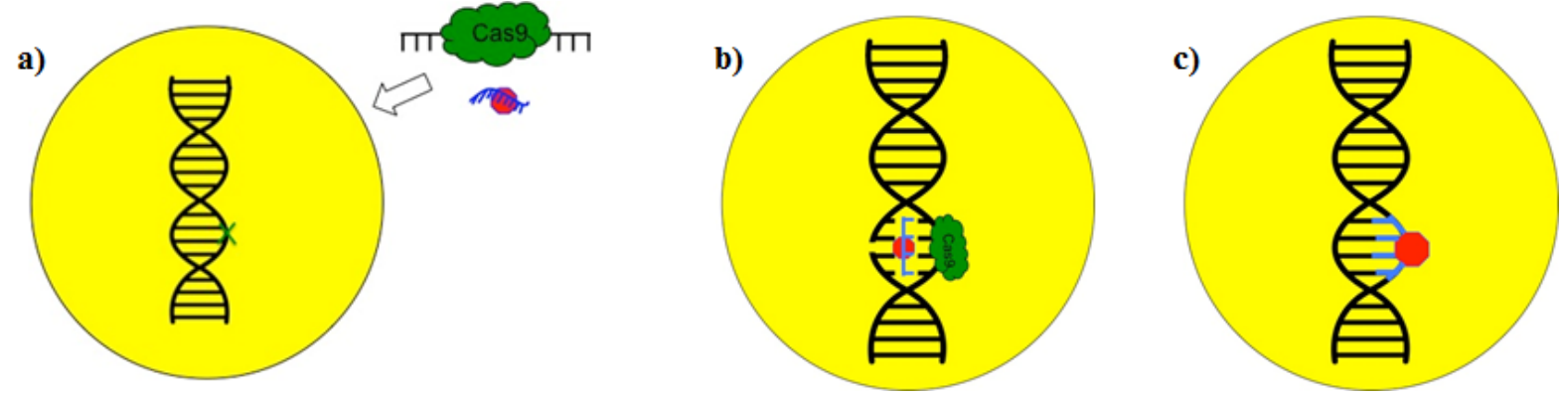

Figure 2: Representation of the mechanism of genome editing using CRISPR-Cas9. a) Cells are transfected with Cas9 protein (green) bound to crRNA to serve as a guide to the genomic location. Cells are also transfected with a DNA oligo complementary to the region of interest and containing the desired mutation, in this case, a nonsense mutation. b) The Cas9 protein binds to the region of interest and creates a doublestranded break. The DNA oligo serves as a template for homologous DNA repair. c) The nonsense mutation has been incorporated into the gene of interest.

Building upon the results of Wang et al, Long and colleagues hypothesized that they could correct the PTC in the Dmd gene in mdx mouse zygotes in order to prevent muscular dystrophy [34]. crRNAs approximately $20 \mathrm{bp}$ in length were designed as the guide strand to target the PTC locus in exon 23 and later injected into the zygotes along with $\operatorname{Cas} 9$ mRNA and a template with the correct Dmd sequence to facilitate HDR. The zygotes were implanted into a surrogate female and the resulting mice were genetic mosaics with $2-100 \%$ correction in the Dmd gene, either via HDR or NHEJ, the latter of which created a small deletion of bases surrounding the PTC, but still lead to fulllength dystrophin expression [34]. Myofibers from mice with varying levels of restored dystrophin function $(17 \%, 41 \%$, and $83 \%)$ were studied and none showed the characteristic mdx phenotype. As well, serum CK levels were comparable to controls and muscle performance tests showed that the CRISPR mice had enhanced muscle performance compared to the mdx mice. Long et al. concluded that their results could be adapted for human somatic cell therapy if gene delivery systems are improved and if the efficiency of HDR could be increased, because terminally differentiated cells, including myocytes in myofibers and cardiomyocytes, lack homologous recombination proteins [35].

The application of CRISPR technology to correct deleterious mutations is also potentially useful in regenerative medicine. In essence, somatic cells such as cultured skin fibroblasts, or blood cells that can be obtained noninvasively from patients, are reprogrammed using transcriptional factors c-Myc, Klf4, Oct4, and Sox2 (MKOS) to become induced pluripotent stem cells (iPSCs) [36]. Upon correction of the disease-causing mutation(s) using CRISPR technology, iPSCs with the wild type sequence will then be differentiated to the desired cell types, e.g. hematopoietic stem cells for treatment of thalassemia and dopaminergic neurons for treatment of Parkinson's disease, and infused/transplanted back to the patient. Recently, we have generated iPSCs from cultured skin fibroblasts from patients with type 2 Gaucher disease [37] and Sanfilippo syndrome B [38] by using a Sendai virus vector carrying the MKOS factors for a CRISPR-mediated DNA repair study. The Sendai virus is a non-integrating virus and thus circumvents the pitfalls of insertional mutagenesis and oncogenesis associated with retroviral and lentiviral reprogramming vectors [39].

\section{Determining protein threshold}

Another impediment to the 'cure-all' promise of these drugs/ treatments is the extreme heterogeneity in the level of protein/enzyme activity required to ameliorate disease symptoms. For recessive diseases, heterozygote carriers have $50 \%$ of normal protein function and are usually asymptomatic [40]; but it is largely variable as to where the real disease threshold is. For example, patients with Mucopolysaccharidosis type I - Hurler's (MPS-I-H) syndrome only require $0.4-1 \%$ of normal $\alpha$-L-iduronidase enzyme activity to alleviate symptoms [41], whereas patients with protein $\mathrm{C}$ deficiency require at least $50 \%$ protein function or else they will express the thrombotic disease [42]. Unfortunately, many diseases have not been characterized this way; it is simply mentioned that due to recessive inheritance, heterozygote carriers (assumed to have $50 \%$ of normal protein/enzyme activity) are unaffected. In future, it will be paramount that the protein threshold of every disease attributed to a premature stop codon be determined in order to devise personal treatment options for a genotypic subset of patients. For example, if a patient has MPS-I-H, PTC suppression therapy alone may be adequate to correct the disease phenotype, by virtue of the low threshold $(0.4-1 \%$ of normal iduronidase). However, if a patient has DMD, it would be advisable that the patient combines stop codon read-through therapies with other symptom management strategies.

\section{Conclusion}

Current PTC suppression therapies have achieved modest increases in protein function; however, these increases are not sufficient to have a therapeutic benefit at present. For example, Malik et al. treated 
sixteen boys with DMD with gentamicin for six months and one patient showed the greatest increase in dystrophin expression in muscle from $4.5 \%$ to $15.44 \%$ [43], Unfortunately, at least $20-30 \%$ of normal dystrophin is required to attenuate DMD symptoms [44]. In order for these treatments to be adapted to a clinical setting, efficiency of read-through must increase, toxicity of the proposed compounds must decrease, delivery mechanisms for gene therapy must improve, and the creation of animal models for the study of these very specific mutational events must be more cost effective and efficient.

\section{Acknowledgement}

This work is supported by an operating grant from the Sanfilippo Children's Research Foundation to F.Y.M.C and a research fellowship from the Natural Sciences and Engineering Research Council of Canada to K.A.T.

\section{References}

1. Crick FHC (1956) On protein synthesis. Symp Soc Exp Biol 12: 139-163.

2. Popp MW, Maquat LE (2013) Organizing principles of mammalian nonsense-mediated mRNA decay. Annu Rev Genet 47: 139-165.

3. Stenson PD, Mort M, Ball EV, Shaw K, Phillips A, et al. (2014) The Human Gene Mutation Database: Building a comprehensive mutation repository for clinical and molecular genetics, diagnostic testing and personalized genomic medicine. Hum Genet 133: 1-9.

4. Keeling KM, Xue X, Gunn G, Bedwell DM (2014) Therapeutics based on stop codon readthrough. Annu Rev Genomics Hum Genet 15: 371-394.

5. Temple GF, Dozy AM, Roy KL, Kan YW (1982) Construction of a functional human suppressor tRNA gene: an approach to gene therapy for beta-thalassaemia. Nature 296: 537-540.

6. Elmezayen AD, Kotb SM, Sadek NA, Abdalla EM (2015) $\hat{I}^{2}$-Globin Mutations in Egyptian Patients With $\hat{\mathrm{I}}^{2}$-Thalassemia. Lab Med 46: 8-13.

7. Kiselev AV, Ostapenko OV, Rogozhkina EV, Kholod NS, Seit Nebi AS, et al. (2002) [Suppression of nonsense mutations in the Dystrophin gene by a suppressor tRNA gene]. Mol Biol (Mosk) 36: 43-47.

8. Howe SJ, Mansour MR, Schwarzwaelder K, Bartholomae C, Hubank M, et al. (2008) Insertional mutagenesis combined with acquired somatic mutations causes leukemogenesis following gene therapy of SCID-X1 patients. J Clin Invest 118: 3143-3150.

9. Seregin SS, Amalfitano A (2010) Improving adenovirus based gene transfer: strategies to accomplish immune evasion. Viruses 2: 2013-2036.

10. Shmeeda H, Amitay Y, Tzemach D, Gorin J, Gabizon A (2013) Liposome encapsulation of zoledronic acid results in major changes in tissue distribution and increase in toxicity. J Control Release 167: 265-275.

11. Yadavalli SS, Ibba M (2013) Selection of tRNA charging quality control mechanisms that increase mistranslation of the genetic code. Nucleic Acids Res 41: 1104-1112.

12. Loh PG, Song H (2010) Structural and mechanistic insights into translation termination. Curr Opin Struct Biol 20: 98-103.

13. Scheunemann AE, Graham WD, Vendeix FA, Agris PF (2010) Binding of aminoglycoside antibiotics to helix 69 of 23 rRNA. Nucleic Acids Res 38: 3094-3105.

14. Eustice DC, Wilhelm JM (1984) Fidelity of the eukaryotic codonanticodon interaction: interference by aminoglycoside antibiotics. Biochemistry 23: 1462-1467.

15. Lee HL, Dougherty JP (2012) Pharmaceutical therapies to recode nonsense mutations in inherited diseases. Pharmacol Ther 136: 227-266.

16. Barton-Davis ER, Cordier L, Shoturma DI, Leland SE, Sweeney HL (1999) Aminoglycoside antibiotics restore dystrophin function to skeletal muscles of mdx mice. J Clin Invest 104: 375-381.

17. Yiu EM, Kornberg AJ (2008) Duchenne muscular dystrophy. Neurol India 56: 236-247.
18. Wagner KR, Hamed S, Hadley DW, Gropman AL, Burstein AH, et al. (2001) Gentamicin treatment of Duchenne and Becker muscular dystrophy due to nonsense mutations. Ann Neurol 49: 706-711.

19. Kuschal C, Khan SG, Enk B, DiGiovanna JJ, Kraemer KH (2015) Readthrough of stop codons by use of aminoglycosides in cells from xeroderma pigmentosum group C patients. Exp Dermatol 24: 296-297.

20. Welch EM, Barton ER, Zhuo J, Tomizawa Y, Friesen WJ, et al. (2007) PTC124 targets genetic disorders caused by nonsense mutations. Nature 447: 87-91.

21. (2000) PTC Therapeutics. Phase $2 \mathrm{~b}$ extension study of ataluren (PTC124) in Duchenne/Becker muscular dystrophy (DMD/BMD). In: ClinicalTrials.gov [Internet]. Bethesda (MD): National Library of Medicine (US).

22. Auld DS, Thorne N, Maguire WF, Inglese J (2009) Mechanism of PTC124 activity in cell-based luciferase assays of nonsense codon suppression. Proc Natl Acad Sci U S A 106: 3585-3590.

23. Auld DS, Lovell S, Thorne N, Lea WA, Maloney DJ, et al. (2010) Molecular basis for the high-affinity binding and stabilization of firefly luciferase by PTC124. Proc Natl Acad Sci U S A 107: 4878-4883.

24. Gonzalez-Hilarion S, Beghyn T, Jia J, Debreuck N, Berte G, et al. (2012) Rescue of nonsense mutations by amlexanox in human cells. Orphanet J Rare Dis 7: 58.

25. Bedwell DM, Wang D, Welch EM, Keeling KM (2015) The nonsense suppression drug PTC124 restored alpha-I-iduronidase activity and reduces glycosaminoglycan accumulation in MPS IH mice carrying the Idua-W402X mutation. Molec Genet Metab 114: S20.

26. Bartolomeo R, Polishchuk EV, Volpi N, Polishchuk RS, Auricchio A (2013) Pharmacological read-through of nonsense ARSB mutations as a potential therapeutic approach for mucopolysaccharidosis VI. J Inherit Metab Dis 36: 363-371.

27. Drake KM, Dunmore BJ, McNelly LN, Morrell NW, Aldred MA (2013) Correction of nonsense BMPR2 and SMAD9 mutations by ataluren in pulmonary arterial hypertension. Am J Respir Cell Mol Biol 49: 403-409.

28. Buck NE, Wood LR, Hamilton NJ, Bennett MJ, Peters HL (2012) Treatment of a methylmalonyl-CoA mutase stopcodon mutation. Biochem Biophys Res Commun 427: 753-757.

29. Konstan M, Accurso F, De Boeck K, Kerem E, Rowe S, et al. (2012) Targeting class I mutations: update on ataluren as a promising treatment for nonsense mutation cystic fibrosis. Pediatr Pulmonol 47: 108-109.

30. Sinclair GB, Jevon G, Colobong KE, Randall DR, Choy FY, et al. (2007) Generation of a conditional knockout of murine glucocerebrosidase: utility for the study of Gaucher disease. Mol Genet Metab 90: 148-156.

31. Wiedenheft B, Sternberg SH, Doudna JA (2012) RNA-guided genetic silencing systems in bacteria and archaea. Nature 482: 331-338.

32. Cong L, Ran FA, Cox D, Lin S, Barretto R, et al. (2013) Multiplex genome engineering using CRISPR/Cas systems. Science 339: 819-823.

33. Wang H, Yang H, Shivalila CS, Dawlaty MM, Cheng AW, et al. (2013) One-step generation of mice carrying mutations in multiple genes by CRISPR/Cas-mediated genome engineering. Cell 153: 910-918.

34. Long C, McAnally JR, Shelton JM, Mireault AA, Bassel-Duby R, et al. (2014) Prevention of muscular dystrophy in mice by CRISPR/Cas9mediated editing of germline DNA. Science 345: 1184-1188.

35. Hsu PD, Lander ES, Zhang F (2014) Development and applications of CRISPR-Cas9 for genome engineering. Cell 157: 1262-1278.

36. Takahashi K, Tanabe K, Ohnuki M, Narita M, Ichisaka T, et al. (2007) Induction of pluripotent stem cells from adult human fibroblasts by defined factors. Cell 131: 861-872.

37. Campbell TN, Jack AT, Choy FYM (2015) 'Gaucher disease and associated dementia' in Diet and Nutrition in Dementia and Cognitive Decline. Chapter 7: 62-75. Elsevier-Academic Press, London.

38. Turner KA, Choy FYM (2015) Sanfilippo syndrome type B: Reprogramming cultured skin fibroblasts to induced pluripotent stem cells using non-integrating Sendai virus vector. Molec Genet Metab 114: S118. 
Citation: Turner KA, Choy FYM (2015) Treatment of Nonsense Mutations Using Stop Codon Read-through Therapeutics and Creation of Animal Models Using CRISPR-Cas9. J Mol Genet Med 9: 172. doi:10.4172/1747-0862.1000172

Page 6 of 6

39. Fusaki N, Ban H, Nishiyama A, Saeki K, Hasegawa M (2009) Efficient induction of transgene-free human pluripotent stem cells using a vector based on Sendai virus, an RNA virus that does not integrate into the host genome. Proc Jpn Acad Ser B Phys Biol Sci 85: 348-362.

40. Bell CJ, Dinwiddie DL, Miller NA, Hateley SL, Ganusova EE, et al. (2011) Carrier testing for severe childhood recessive diseases by next-generation sequencing. Sci Transl Med 3: 65ra4.

41. Bunge S, Clements PR, Byers S, Kleijer WJ, Brooks DA, et al. (1998) Genotype-phenotype correlations in mucopolysaccharidosis type I using enzyme kinetics, immunoquantification and in vitro turnover studies. Biochim Biophys Acta 1407: 249-256.
42. Griffin JH, Evatt B, Zimmerman TS, Kleiss AJ, Wideman C (1981) Deficiency of protein $\mathrm{C}$ in congenital thrombotic disease. J Clin Invest 68: 1370-1373.

43. Malik V, Rodino-Klapac LR, Voillet L (2010) Aminoglycoside-induced mutation suppression (stop codon readthrough) as a therapeutic strategy for Duchenne muscular dystrophy. Ther Adv Neurol Disord 3: 379-389.

44. Chamberlain J (1997) Dystrophin levels required for genetic correction of Duchenne muscular dystrophy. Basic Appl Myol 7: 251-255. 